Article

\title{
Midpalatal Suture Density as a Function of Sex and Growth-Pattern-Related Variability via CBCT Evaluations of 392 Adolescents Treated with a Rapid Maxillary Expander Appliance
}

\author{
Monica Macrì * (D), Elena Toniato, Giovanna Murmura, Giuseppe Varvara $\mathbb{D}^{\mathbb{D}}$ and Felice Festa
}

check for updates

Citation: Macrì, M.; Toniato, E.; Murmura, G.; Varvara, G.; Festa, F. Midpalatal Suture Density as a Function of Sex and Growth-Pattern-Related Variability via CBCT Evaluations of 392

Adolescents Treated with a Rapid Maxillary Expander Appliance. Appl. Sci. 2022, 12, 2221. https://doi.org/ 10.3390/app12042221

Academic Editor: Andrea Scribante

Received: 7 January 2022

Accepted: 14 February 2022

Published: 21 February 2022

Publisher's Note: MDPI stays neutral with regard to jurisdictional claims in published maps and institutional affiliations.

Copyright: () 2022 by the authors. Licensee MDPI, Basel, Switzerland. This article is an open access article distributed under the terms and conditions of the Creative Commons Attribution (CC BY) license (https:// creativecommons.org/licenses/by/ $4.0 /)$.

\author{
Department of Innovative Technologies in Medicine \& Dentistry, University "G. D'Annunzio" of Chieti-Pescara, \\ Via dei Vestini, 66100 Chieti, Italy; elena.toniato@unich.it (E.T.); giovanna.murmura@unich.it (G.M.); \\ gvarvara@unich.it (G.V.); ffesta@unich.it (F.F.) \\ * Correspondence: m.macri@unich.it
}

\begin{abstract}
Background and objectives. This paper aimed to evaluate the changes in mean bone density values of the midpalatal suture (MPS) in 392 young patients treated with a rapid palatal expander (RPE) appliance, depending on sex and vertical and sagittal skeletal patterns. Materials and Methods. Evaluations were performed using a low-dose protocol for cone beam computed tomography scans at T0 (preoperative) and T1 (1 year after the beginning of the therapy). The region of interest was used to calculate bone density in Hounsfield units (HU) for the area between the maxillary incisors. Results. CBCT scan data of 196 females and 196 males (mean age of 11.7 years) showed homogeneous and similar density values of the MPS at T0 (550.17-563.70 HU) and T1 (541.92-553.85 HU). Class III skeletal individuals showed significantly higher BD than the Class II group at T0, but not at T1. Females showed significant and substantially higher BD than males at T0 and T1. No significant differences were found between the other groups and between the two time points in terms of the bone density values of the MPS. Conclusions. Females and the Class III group showed significantly higher bone density values than males and Class II, respectively. No statistically significant differences were found from T0 to T1 in any groups, suggesting that a similar rate of suture reorganization occurs after the use of an RPE, causing reorganization and bone deposition along the MPS.
\end{abstract}

Keywords: rapid palatal expander; midpalatal suture; bone density; cone beam computed tomography; facial patterns; skeletal growth pattern

\section{Introduction}

Maxillary transverse deficiency (MTD, or maxillary hypoplasia) is a common problem that affects the normal development of the maxillofacial complex. Therefore, the early diagnosis and correction of MTD are essential to achieve a normal transverse skeletal relationship between maxilla and mandible [1].

The incidence of MTD ranges from $8.5 \%$ to $22 \%$ [2] in children and adolescents. MTD is usually associated with a unilateral or bilateral crossbite [3], a generalized lack of space in the maxillary arch, and crowding because the jaw is narrow compared with the rest of the craniofacial structures. These conditions can be treated using an RPE as described by Angell (1860) [4]. The RPE appliance is used in growing patients because the ossification of the MPS increases with age and occurs earlier in girls than in boys [5]. In terms of sex, the mean age of ossification of the MPS in girls is 16 years and 18 years in boys, as described by Melsen in 1975 [6]. However, Haas in 1980 reported that MPS fusion occurs at the age of 14-15 in females and 15-16 in males [1]. The maturation stages of the MPS are not directly related to chronological age, as reported in numerous studies $[7,8]$. The hand and wrist 
method (HWM) [7] and cervical vertebrae method (CVM) [8] are the conventional indexes chosen to determine the possibility of maxillary expansion. Angelieri (2013) [9] introduced a new index by observing CBCT images, and suggested that maturation of the MPS can be Classified into five stages (A, B, C, D, E). There are three types of MPS disjunction: RPE (with dental support), MARPE (miniscrew-assisted rapid palatal expansion with skeletal support) [10], and SARPE (surgically-assisted rapid palatal expansion) [11]. MARPE and SARPE are used in fused MPSs or compromised dental support.

MPS fusion Classification using CBCT provides reliable parameters for the clinical decision between conventional (RPE) and unconventional (MARPE, SARPE) methods. According to Angelieri et al., (2013) [9], there are five stages of MPS maturation. Stages A (straight high-density sutural line, with no or little interdigitation) and B (scalloped appearance of the high-density sutural line) are frequently noted up to the age of 13 years; stage C (parallel, scalloped, high-density lines that were close to each other, separated in some areas by small, low-density spaces) is typically observed from 11 to 17 years (rarely in younger or older patients). Patients in stages $\mathrm{D}$ (fusion completed in the palatine bone, with no evidence of a suture) and $\mathrm{E}$ (anterior fusion in the maxilla) might be better treated by SARPE because fusion of the MPS already has occurred.

The introduction of cone beam computed tomography (CBCT) in orthodontics allows a quantitative evaluation of BD using Hounsfield units (HU) [12], and an accurate analysis concerning sagittal and vertical growth patterns, which helps to make the decision about whether to use conventional (RPE) or unconventional maxillary expansion methods (MARPE or SARPE).

The present paper aims to measure mean bone density (BD) values of the midpalatal suture (MPS) in order to predict an accurate estimation of the MPS's response to expansion therapy using a rapid palatal expander (RPE) appliance, as related to sex (males and females), vertical skeletal patterns (hypodivergent, normodivergent, and hyperdivergent), and sagittal skeletal patterns (Class I, Class II, and Class III).

The first null hypothesis predicts no differences between males and females in terms of $\mathrm{BD}$ in the ROI. The second null hypothesis predicts that there are no differences between different vertical skeletal patterns in terms of BD in the ROI. The third null hypothesis predicts no differences between different sagittal skeletal patterns in terms of BD in the ROI. The fourth null hypothesis predicts that there are no differences between the two time points (T0 and $\mathrm{T} 1$ ) in $\mathrm{BD}$ in the ROI.

\section{Normal Material and Methods}

\subsection{Study Design}

In this retrospective study, the CBCT scans of 392 early adolescents aged between 10 and 14 years who were treated with an RPE appliance were analyzed using Dolphin software. The bone density (BD) of the MPS was calculated at two time points: preoperatively (T0) and one year after the beginning of the treatment with the RPE (T1).

\subsection{Setting (Setting, Locations, and Relevant Dates, including Periods of Recruitment, Exposure,} Follow-Up, and Data Collection)

The clinical data were collected from the archives of the Department of Medical, Oral, and Biotechnological Sciences of the University "G. D'Annunzio" in Chieti (from 2016 to 2021). Ethical approval (number 23) was obtained by the Independent Ethics Committee of the hospital of Chieti. This study's protocol was created based on the European Union Good Practice Rules and in line with the Helsinki Declaration.

\subsection{Participants}

Written informed consent was obtained from all patients included in the study.

This retrospective study recruited both sexes: 196 males and 196 females. For vertical skeletal growth pattern differentiation, the subjects were Classified by the Frankfort horizontal line to the mandibular plane angle (hypodivergent $<22^{\circ}, 22^{\circ} \leq$ normodivergent $\leq 28^{\circ}$, 
and $28^{\circ}<$ hyperdivergent) [13]. For the sagittal growth pattern analysis, the participants were divided by the ANB angle (Class III $<0^{\circ}, 0^{\circ} \leq$ Class I $\leq 4^{\circ}$, and $4^{\circ}<$ Class II) [14].

The BD of the MPS was calculated at two time points: preoperatively (T0), and one year after the beginning of the treatment with the RPE (T1). This study was carried out using CBCT with a low-dose protocol [15], and the region of interest (ROI) was used to calculate BD in the area between the maxillary incisors (manually standardized in each CBCT scan).

The inclusion and exclusion criteria are reported in Table 1.

Table 1. Inclusion and exclusion criteria.

\begin{tabular}{|c|c|}
\hline Inclusion Criteria & Exclusion Criteria \\
\hline $\begin{array}{l}\text { age ranged from } 10 \text { to } 14 \text { years (early } \\
\text { adolescence) [16] } \\
\text { presence of a transverse } \\
\text { maxillary deficiency } \\
\text { presence of a unilateral or bilateral } \\
\text { posterior crossbite } \\
\text { presence of a complete CBCT exam at T0 } \\
\text { and T1 } \\
\text { success of the therapy with an } \\
\text { RPE appliance }\end{array}$ & $\begin{array}{ll}\text { - } & \text { lack of any diagnostic data (including } \\
\text { - } & \text { CBCT images at T0 and T1) } \\
- & \text { poor quality CBCT images } \\
- & \text { impacted or missing teeth } \\
- & \text { dentofacial abnormalities } \\
- & \text { skeletal asymmetry } \\
- & \text { any syndromes or pathologies involving } \\
\text { bone metabolism. }\end{array}$ \\
\hline
\end{tabular}

\subsection{Treatment}

All patients in the study underwent transverse maxillary expansion with a banded RPE on dental support: the RPE was initially activated on the chair by performing a complete turn of the Hyrax screw, which corresponds to 4 activations $(1 \mathrm{~mm})$. The patients were instructed to start the RPE at home, twice a day ( $0.5 \mathrm{~mm}$ expansion a day) until a $2 \mathrm{~mm}$ molar transverse overcorrection was achieved (generally for 10-15 days). The mean maximum expansion was $7.25 \pm 1.25 \mathrm{~mm}$. The same RPE was subsequently used as a passive retainer to prevent transverse maxillary relapse for six months, and the screw was locked with a light-cure flow composite. The appliance was removed six months after its last activation. A second CT scan was performed 12 months after the treatment with the RPE using the same parameters and conditions as the previous exam at T0. Before and after treatment, patients underwent pain assessments (via measures of visual analogue scales and muscular palpation tests) [17].

\section{5. $C B C T$}

All CBCT examinations were taken at T0 and T1, and were performed by the Planmeca Promax ${ }^{\circledR}$ 3D MID unit (Planmeca Oy, Helsinki, Finland) according to the low-dose protocol [15] with these parameters: an acquisition time of $15 \mathrm{~s}, 80 \mathrm{kVp}, 5 \mathrm{~mA}, 35$ microSievert $(\mu \mathrm{Sv})$, and a field of view $(\mathrm{FOV})$ of $240 \times 190$.

The patients' CBCTs were performed with the head oriented according to the natural head position (NHP); the patients were in a sitting position with the back perpendicular to the floor as much as possible. The head was stabilized with ear rods in the external auditory meatus. The patients were instructed to look into their own eyes in a mirror $1.5 \mathrm{~m}$ in front of them to obtain NHP. The NHP is a physiological and reproducible posture defined for the morphological analysis described in orthodontic and anthropological literature. The 3D image of the cranium was oriented in the Dolphin software according to NHP posture before taking cephalometric measurements. The NHP orientation was carried out by the widgets present in Dolphin; hard- and soft-tissue views were checked for orientation in the software by visualizing the head from the front, right, and left sides. In the NHP, there are three reference planes perpendicular to each other, which are identified in the software for the patients' cephalometric measurements (Figure 1). 


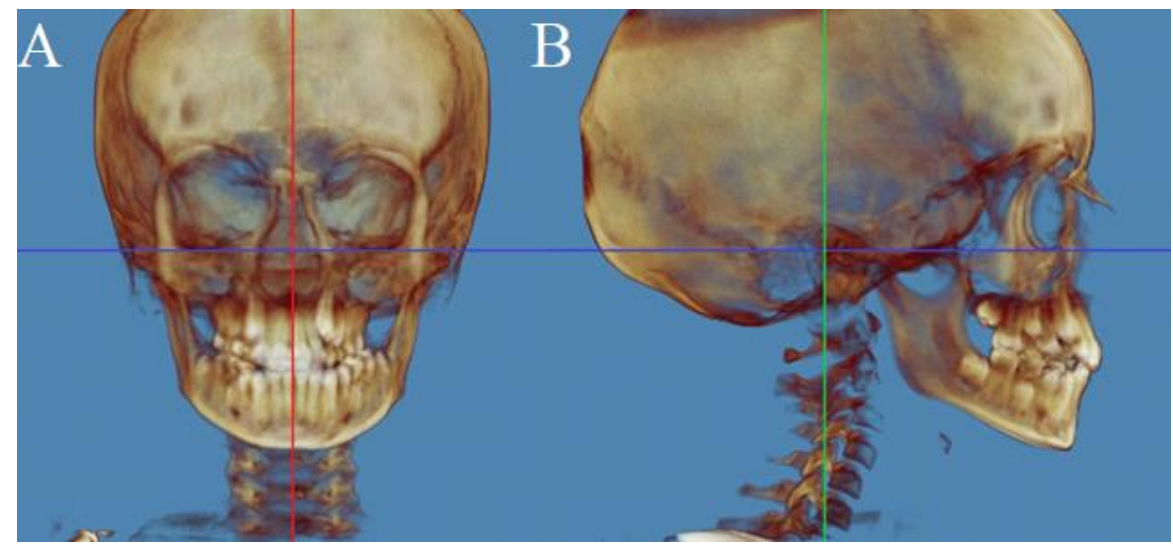

Figure 1. Spatial orientation of the CBCT scans according to the natural head position (NHP). The frontal view scan (Figure 1A) and lateral view scan (Figure 1B) are from the same patient. (A) The red line in the coronal orientation coincides with the mid-sagittal plane (MSP), a plane perpendicular to plane $\mathrm{FH}$ and passing through Crista Galli $(\mathrm{Cg})$ and Basion $(\mathrm{Ba})$ points. $(\mathrm{B})$ The blue line in the sagittal orientation coincides with the Frankfurt plane (FH), a plane passing through Orbital (Or) and Porion (Po) points; the green line coincides with the anteroposterior (PO) plane, perpendicular to the FH and MSP, passing through the Porion.

1. The transverse plane coincides with the Frankfurt plane (FH), a plane passing through two points: Orbital (Or) and Porion (Po);

2. The sagittal plane coincides with the mid-sagittal plane (MSP), a plane perpendicular to plane $\mathrm{FH}$ and passing through two points: Crista Galli $(\mathrm{Cg})$ and Basion $(\mathrm{Ba})$;

3. The coronal plane coincides with the anteroposterior (PO) plane, perpendicular to the FH and MSP, passing through the right and left Porion.

The examiners manually defined the ROI and used it to calculate the bone density of the MPS in Hounsfield units (HU), twice. The ROI [18] for all patients is the delimited area (Figure 2):

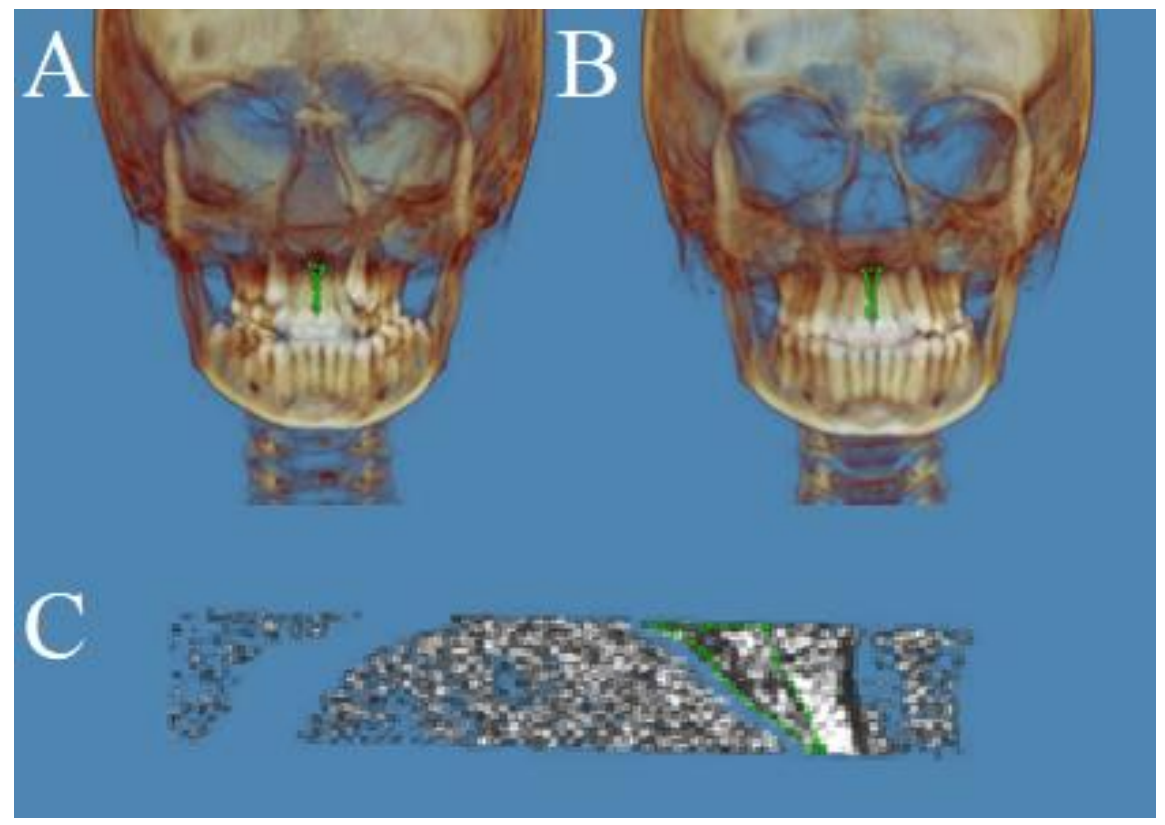

Figure 2. The region of interest (ROI) was selected at T0 (A) and T1 (B), as shown in green. The ROI is delimited superiorly by the upper central incisors' apex, laterally by the medial root surface of the upper central incisors, and inferiorly by the cementoenamel junction $(\mathbf{A}, \mathbf{B})$. The ROI is delimited posteriorly by the anterior edge of the nasopalatine foramen $(\mathbf{C})$. 
- $\quad$ superiorly by the upper central incisors' apex

- $\quad$ laterally by the upper central incisors' medial root surface

- $\quad$ inferiorly by the cementoenamel junction

- posteriorly by the anterior edge of the nasopalatine foramen

Sex and vertical and sagittal skeletal patterns were used to categorize the sample into eight groups as reported in Table 2:

Table 2. The groups of participants according to sex and skeletal pattern.

\begin{tabular}{|c|c|c|}
\hline Sex & Vertical Skeletal Pattern & Sagittal Skeletal Pattern \\
\hline $\begin{array}{ll}\text { - } & \text { Males ( } n 196,50 \% \text { of } \\
392 \text { patients) } \\
\text { - } \quad \text { Females ( } n 196,50 \% \text { of } \\
392 \text { patients) }\end{array}$ & $\begin{array}{ll}\text { - } & \text { Hypodivergent }(n 122, \\
31.1 \% \text { of } 392 \text { patients }) \\
\text { - }\end{array} \quad \begin{array}{l}\text { Normovergent }(n 141, \\
36.0 \% \text { of } 392 \text { patients) } \\
-\quad\end{array} \quad \begin{array}{l}\text { Hyperdivergent }(n 129, \\
32.9 \% \text { of } 392 \text { patients })\end{array}$ & $\begin{array}{ll}\text { - } & \text { Class I }(n 139,35.5 \% \text { of } \\
& 392 \text { patients }) \\
-\quad & \text { Class II }(n 106,27.0 \% \text { of } \\
& 392 \text { patients }) \\
\text { - } & \text { Class III }(n 147,37.5 \% \text { of } \\
& 392 \text { patients })\end{array}$ \\
\hline
\end{tabular}

Each measurement was performed twice by the same examiners at the first (T0) and second (T1) CBCT scan for all patients.

\subsection{Error Method}

All the CBCT images were randomly selected and analyzed to evaluate the reliability of this study. To validate the repeatability and reproducibility of a quantitative evaluation of $\mathrm{BD}$ and to assess intraoperator and interoperator errors, the CBCT data of the patients were processed by two different operators, twice; the Wilcoxon signed-rank test evaluated changes in the calculated BD between the first and second measurements for each operator. No significant differences were observed between the two measurements for the BD for both operators.

The reliability between operators 1 and 2 was determined with intra-Class correlation coefficients (ICC). The mean BD of the MPS values displayed excellent reliability with inter-rater ICC's between operators 1 and 2.

\subsection{Statistical Analysis}

Simple descriptive statistics were used to summarize the data, as reported in Table 3. Means and standard deviations $(\sigma)$ were computed for BD at T0 and T1 for each category of patients: males, females, hypodivergent, normodivergent, hyperdivergent, Class I, Class II, and Class III. A one-sample Kolmogorov-Smirnov test was used to test the normality of BD for the various categories (sex and vertical and sagittal skeletal patterns) [19]. The values of the K-S test statistic were $0.14(p=0)$ at T0 and $0.23(p=0.70)$ at T1, so the assumption of normality was not violated because the data do not differ significantly from what is normally distributed. The values of density at the same time point (means, standard deviations, and $n s$ ) were analyzed by an independent-samples $t$-test in terms of sex (male and female), vertical skeletal pattern (hypodivergent vs. hyperdivergent, hypodivergent vs. normodivergent, normodivergent vs. hyperdivergent), and sagittal skeletal pattern (Class I vs. Class II, Class I vs. Class III, Class II vs. Class III). Statistical analyses were performed using GraphPad Prism software (GraphPad Software Inc., San Diego, CA, USA). A Friedman repeated-measures ANOVA computed the changes in density from T0 to T1 on ranks followed by a Tukey post-hoc test. The level of significance was set at $p<0.05$. 
Table 3. The mean bone density (BD) of the MPS according to sex and skeletal pattern (HU).

\begin{tabular}{|c|c|c|c|c|c|c|c|c|c|}
\hline \multicolumn{10}{|c|}{ Mean $(\mu)$ and Standard Deviation $(\sigma)$} \\
\hline & & \multicolumn{2}{|c|}{ Sex } & \multicolumn{3}{|c|}{ Vertical Skeletal Pattern } & \multicolumn{3}{|c|}{ Sagittal Skeletal Pattern } \\
\hline & & Males & Females & $\begin{array}{c}\text { Hypo- } \\
\text { Divergent }\end{array}$ & $\begin{array}{c}\text { Normo- } \\
\text { Divergent }\end{array}$ & $\begin{array}{c}\text { Hyper- } \\
\text { Divergent }\end{array}$ & Class-III & Class-I & Class-II \\
\hline & n & 196 & 196 & 122 & 141 & 129 & 147 & 139 & 106 \\
\hline \multirow{2}{*}{ T0 } & $\mu(\mathrm{Hu})$ & 550.17 & 561.35 & 551.08 & 556.52 & 552.84 & 563.70 & 558.72 & 555.04 \\
\hline & $\sigma$ & 27.12 & 24.33 & 26.03 & 24.72 & 24.10 & 25.91 & 26.18 & 23.32 \\
\hline \multirow{2}{*}{ T1 } & $\mu(\mathrm{Hu})$ & 541.92 & 550.25 & 545.23 & 541.16 & 543.72 & 553.85 & 552.29 & 550.66 \\
\hline & $\sigma$ & 25.09 & 24.21 & 25.96 & 25.24 & 24.82 & 26.16 & 23.76 & 26.89 \\
\hline
\end{tabular}

\section{Results}

CBCT scan data for 392 patients (196 females and 196 males) who had a mean age of 11.7 years (range from 10 to 13.9 years) and underwent RPE were included in the study. The bone densities of the MPS in HU for different categories (sex and skeletal pattern) and for different time points (T0 and $\mathrm{T} 1$ ) are shown in Table 3.

\subsection{Sex Comparison}

At T0, the two-tailed $p$-value was less than 0.0001. By conventional criteria, this difference is extremely statistically significant $(p>0.05)$. The mean bone density of the MPS of females minus males equals 11.1800 ( $95 \%$ confidence interval, $t=4.2960, \mathrm{df}=390$, standard error of difference $=2.602$ ). At T1, the two-tailed $p$-value equaled 0.0009. By conventional criteria, this difference is extremely statistically significant $(p>0.05)$. The mean bone density of the MPS of females minus males equaled 8.3300 (95\% confidence interval, $\mathrm{t}=3.3448, \mathrm{df}=390$, standard error of difference $=2.490)$. The mean bone density of the MPS ( $550.17 \pm 27.12 \mathrm{HU}$ at T0, $541.92 \pm 25.09 \mathrm{HU}$ at T1) in the group of males (196 subjects) was significantly lower than that $(561.35 \pm 24.33 \mathrm{HU}$ at T0, $545.23 \pm 25.95 \mathrm{HU}$ at T1 $)$ in the group of females (196 subjects).

\subsection{Hypodivergent vs. Normodivergent}

At T0, the two-tailed $p$-value equaled 0.1467. By conventional criteria, this difference is not statistically significant $(p<0.05)$. The mean bone density of the MPS of hypodivergent minus normodivergent equaled 4.5600 (95\% confidence interval, $\mathrm{t}=1.4556, \mathrm{df}=261$, standard error of difference $=3.133$ ).

At T1, the two-tailed $p$-value equaled 0.1992. By conventional criteria, this difference is not considered to be statistically significant $(p<0.05)$. The mean bone density of the MPS of hypodivergent minus normodivergent equaled 4.0700 ( $95 \%$ confidence interval, $\mathrm{t}=1.2870, \mathrm{df}=261$, standard error of difference $=3.162$ ). The mean bone density of the MPS (551.08 $\pm 26.03 \mathrm{HU}$ at T0, $545.23 \pm 25.96 \mathrm{HU}$ at T1) in the group of hypodivergent (122 subjects) was slightly higher than that $(546.52 \pm 24.72 \mathrm{HU}$ at T0, $541.16 \pm 25.24 \mathrm{HU}$ at T1) in the group of normodivergent (141 subjects), but was not statistically significant.

\subsection{Hyperdivergent vs. Normodivergent}

At T0, the two-tailed $p$-value equaled 0.0346 . By conventional criteria, this difference is not considered to be statistically significant. The mean bone density of the MPS of hyperdivergent minus normodivergent equaled 6.3200 (95\% confidence interval, $t=2.1237$, $\mathrm{df}=268$, standard error of difference $=2.976$ ). At T1, the two-tailed $p$-value equaled 0.4022 . By conventional criteria, this difference is not considered to be statistically significant. The mean bone density of the MPS of hyperdivergent minus normodivergent equaled 2.5600 ( $95 \%$ confidence interval, $\mathrm{t}=0.8391, \mathrm{df}=268$, standard error of difference $=3.051$ ). The mean bone density of the MPS (552.84 $\pm 24.10 \mathrm{HU}$ at T0, $543.72 \pm 22.25 \mathrm{HU}$ at T1) in the group of hyperdivergent (129 subjects) was slightly higher than that $(546.52 \pm 23.39 \mathrm{HU}$ at 
$\mathrm{T} 0,542.39 \pm 25.24 \mathrm{HU}$ at T1) in the group of normodivergent (141 subjects), but was not statistically significant.

\subsection{Hypodivergent vs. Hyperdivergent}

At $\mathrm{T} 0$, the two-tailed $p$-value equaled 0.5786. By conventional criteria, this difference is not considered to be statistically significant. The mean bone density of the MPS of hyperdivergent minus hypodivergent equaled 1.7600 ( $95 \%$ confidence interval, $\mathrm{t}=0.5562$, $\mathrm{df}=249$, standard error of difference $=3.164$ ).

At T1, the two-tailed $p$-value equaled 0.6335 . By conventional criteria, this difference is not statistically significant $(p<0.05)$. The mean bone density of the MPS of hyperdivergent minus hypodivergent equaled 1.5300 (95\% confidence interval, $\mathrm{t}=0.4773, \mathrm{df}=249$, standard error of difference $=3.205)$. The mean bone density of the MPS $(552.84 \pm 24.10 \mathrm{HU}$ at T0, $543.72 \pm 24.82 \mathrm{HU}$ at T1) in the group of hyperdivergent (129 subjects) was slightly lower than that $(551.08 \pm 26.03 \mathrm{HU}$ at T0, $545.23 \pm 25.24 \mathrm{HU}$ at T1) in the group of hypodivergent (122 subjects), but was not statistically significant.

\subsection{Class I vs. Class II}

At T0, the two-tailed $p$-value equaled 0.2545 . By conventional criteria, this difference is not statistically significant $(p<0.05)$. The mean bone density of the MPS of Class I minus Class II equaled 3.6800 ( $95 \%$ confidence interval, $\mathrm{t}=1.1422$, $\mathrm{df}=243$, standard error of difference $=3.222$ ).

At T1, the two-tailed $p$-value equaled 0.6158 . By conventional criteria, this difference is not statistically significant $(p<0.05)$. The mean bone density of the MPS of Class I minus Class II equaled 1.6300 (95\% confidence interval, $\mathrm{t}=0.5024, \mathrm{df}=243$, standard error of difference $=3.244)$. The mean bone density of the MPS $(558.72 \pm 26.18 \mathrm{HU}$ at T0, $552.29 \pm 23.27 \mathrm{HU}$ at T1) in the group of Class I (139 subjects) was slightly higher than that $(555.04 \pm 23.32 \mathrm{HU}$ at T0, $550.66 \pm 26.89 \mathrm{HU}$ at T1) in the group of Class II (106 subjects), but was not statistically significant.

\subsection{Class I vs. Class III}

At T0, the two-tailed $p$-value equaled 0.1071. By conventional criteria, this difference is not statistically significant $(p<0.05)$. The mean bone density of the MPS of Class III minus Class I equaled $4.9800(95 \%$ confidence interval, $\mathrm{t}=1.6164, \mathrm{df}=284$, standard error of difference $=3.081$ ).

At T1, the two-tailed $p$-value equaled 0.5986. By conventional criteria, this difference is not statistically significant $(p<0.05)$. The mean bone density of the MPS of Class III minus Class I equaled 1.5600 (95\% confidence interval, $\mathrm{t}=0.5270, \mathrm{df}=284$, standard error of difference $=2.960)$. The mean bone density of the MPS $(558.72 \pm 26.18 \mathrm{HU}$ at T0, $552.29 \pm 23.27 \mathrm{HU}$ at T1) in the group of Class I (139 subjects) was slightly higher than that $(563.70 \pm 25.91 \mathrm{HU}$ at T0, $553.85 \pm 26.16 \mathrm{HU}$ at T1) in the group of Class III (147 subjects), but was not statistically significant.

\subsection{Class II vs. Class III}

At T0, the two-tailed $p$-value was less than 0.0067. By conventional criteria, this difference is considered very statistically significant $(p>0.05)$. The mean bone density of the MPS of Class III minus Class II equaled 8.6600 (95\% confidence interval, $\mathrm{t}=2.7339$, $\mathrm{df}=251$, standard error of difference $=3.168$ ) .

At T1, the two-tailed $p$-value equaled 0.3451. By conventional criteria, this difference is not statistically significant $(p<0.05)$. The mean bone density of the MPS of Class III minus Class II equaled 3.1900 (95\% confidence interval, $\mathrm{t}=0.9459, \mathrm{df}=251$, standard error of difference $=3.373)$. The mean bone density of the MPS $(563.70 \pm 25.91 \mathrm{HU}$ at T0, $553.85 \pm 26.16 \mathrm{HU}$ at T1) in the group of Class III (147 subjects) was significantly higher than that $(555.04 \pm 23.32 \mathrm{HU}$ at T0, $550.66 \pm 26.89 \mathrm{HU}$ at T1) in the group of Class II (106 subjects). 


\subsection{T0 vs. T1}

No statistically significant differences were found between the two time points (from T0 to T1) in any groups $(p<0.05)$.

\section{Discussion}

The purpose of this paper was to evaluate RPE effects in terms of BD of the MPS in growing patients (10-14 years). A low-dose CBCT protocol was used for the better identification of landmarks and to reduce the radiation exposure of the patients. The ROI in the MPS showed a lack of statistically significant differences from T0 (preoperative) to T1 (one year after the beginning of the therapy). According to Lione et al., (2013) [20], who found similar results between T0 (preoperative) and six months after the beginning of RPE, they also found a significant reduction in density between $\mathrm{T} 0$ and the end of the active expansion phase (14 days after T0), which was caused by the orthopaedic forces of RPE that determined the lateral displacement of the two hemi-maxillae, as confirmed by Fastuca et al., (2020) [21].

The RPE was kept in place as a passive retainer to avoid transverse maxillary relapse and to maintain the two halves of the maxilla separately while mineralization of the MPS increases.

As ageing increases, the degree of ossification of the MPS increases; the impact of the RPE was mainly on tipping the teeth after puberty. CBCT evaluation of the MPS is highly recommended before the use of the RPE [5], in order to avoid the failure of maxillary expansion in terms of a lack of increase in transverse width, and in terms of adverse effects such as periodontal attachment loss, increased mobility, uncontrolled tipping, root reabsorption, necrosis, and vestibular fenestration [22].

Females showed significantly higher BD of the MPS than males, and the first null hypothesis was rejected.

No difference between different vertical skeletal patterns (hypodivergent vs. normodivergent, hyperdivergent vs. normodivergent, hypodivergents vs. hyperdivergent) and between BD values before (T0) and after RPE (T1) was found, and thus the second and fourth null hypotheses were not rejected. Similar results were found in the literature by Chae [17].

Class III skeletal individuals showed a significantly higher BD values than the Class II group at T0, but not at T1. No differences between other sagittal skeletal pattern groups were found, so the third null hypothesis was partially rejected. These results were confirmed in the literature [17].

The MPS showed homogeneous density values before treatment (T0) in each category (550.17 HU, 561.35 HU, 551.08 HU, 546.52 HU, 552.84 HU, 563.70 HU, 558.72 HU, and $555.04 \mathrm{HU})$.

The RPE produced maxillary expansion, and MPS opening was successful in all patients recruited in this retrospective study. In the literature, it is reported that it was possible to open sutures with RPE when BD values of the MPS ranged from 563.3 to 741.7 HU, as confirmed by Lione (2013) [20].

The MPS also showed homogeneous density values after treatment (T1) in each group (541.92 HU, 550.25 HU, 545.23 HU, 541.16 HU, 543.72 HU, 553.85 HU, 552.29 HU, and $550.03 \mathrm{HU})$, and the density values of the MPS at T1 were similar to T0, suggesting that a similar rate of suture reorganization occurs one year after the use of RPE, caused by reorganization and bone deposition along the MPS.

There are two limitations to this study. First, the suture margins' poor resolution was not well defined in the low-dose CBCT protocol. The second limitation was the implementation of the inclusion criteria according to age range (from 10 to 14 years), without distinguishing the patients according to the five stages of MPS maturation described by Angelieri (2013). Further research should be designed to prove the utility of CBCT in the evaluation of the mean bone density of the MPS using Hounsfield units. 


\section{Conclusions}

This retrospective study concluded that:

- $\quad$ the female group showed a significant higher BD value than the male group before the RPE (at T0) and 1 year after the beginning of the RPE therapy (T1)

- $\quad$ no differences were found between different vertical skeletal patterns in BD values of MPS at T0 and T1

- the Class III group showed a significant higher BD value than the Class II group at T0, but not at $\mathrm{T} 1$

- $\quad$ no differences were found between other sagittal skeletal pattern (Class I vs. Class II, Class I vs. Class III) groups in terms of BD values of MPS at T0 and T1

- $\quad$ no differences were found between BD values before and after RPE, because similar results were found between $\mathrm{T} 0$ and $\mathrm{T} 1$;

The maturation stages of the MPS are not directly related to chronological age. In conclusion, CBCT evaluation of the BD of the MPS provides a novel, useful predictor for orthodontic treatment planning, and a reliable predictor of the skeletal response to RPE.

Author Contributions: F.F. and M.M. documented the cases. M.M., E.T. and G.V. conducted a review of the literature. M.M. and G.M. drafted the manuscript. M.M and G.V. analyzed the data. All authors have read and agreed to the published version of the manuscript.

Funding: This research received no external funding.

Institutional Review Board Statement: This study involving human participants was reviewed and approved by ethics approval (number 23) which was obtained from the hospital's Independent Ethics Committee of Chieti.

Informed Consent Statement: Informed consent was obtained from all subjects involved in this study.

Data Availability Statement: Not applicable.

Conflicts of Interest: The authors declare no conflict of interest.

\section{References}

1. Sayar, G.; Kılınç, D.D. Rapid maxillary expansion outcomes according to midpalatal suture maturation levels. Prog. Orthod. 2019, 20, 1-7. [CrossRef] [PubMed]

2. Ömezli, M.M.; Torul, D.; Avc1, T. Persistent Palatal Hypertrophy Associated with Rapid Maxillary Expansion Procedure: Report of a Rare Case. Biomedicine 2020, 10, 49. [CrossRef] [PubMed]

3. Jawale, B. “Wonders of Rapid Maxillary Expansion And Lower Premolar Extractions In Correction of A Skeletal Class Iii Case With Maxllary Deficiency And Mandibular Excess III"-A Case Report on Non-Surgical Orthodontic Camouflage. Saudi J. Oral Dent. Res. 2021, 6, 192-202.

4. Cho, A.-R.; Park, J.H.; Moon, W.; Chae, J.-M.; Kang, K.-H. Short-term effects of microimplant-assisted rapid palatal expansion on the circummaxillary sutures in skeletally mature patients: A cone-beam computed tomography study. Am. J. Orthod. Dentofac. Orthop. 2021, 161, e187-e197. [CrossRef]

5. Vo, H.T.T.; Tran, L.T.K.; Nguyen, H.T. Dental and Skeletal Changes On Cone-Beam Computed Tomography After Rapid Maxillary Expansion Using Rapid Palatal Expander for the Growing Children. Oral Maxillofac. Surg. Cases 2021, 7, 100237. [CrossRef]

6. Betlej, A.; Gandhi, V.; Upadhyay, M.; Allareddy, V.; Tadinada, A.; Yadav, S. Variability of the maxillary suture maturation and density in the subjects with different sex and growth status. Clin. Anat. 2021, 34, 357-364. [CrossRef]

7. Yu, D.; Donghyun, K. Assessment of Midpalatal Suture Maturation By Skeletal Maturity On Hand Wrist Radiographs. J. Korean Acad. Pedtatric Dent. 2021, 48, 31-41. [CrossRef]

8. Baccetti, T.; Franchi, L.; McNamara, J.A. The Cervical Vertebral Maturation (CVM) Method for the Assessment of Optimal Treatment Timing in Dentofacial Orthopedics. Semin. Orthod. 2005, 11, 119-129. [CrossRef]

9. Angelieri, F.; Cevidanes, L.H.; Franchi, L.; Gonçalves, R.; Benavides, E.; McNamara, J.A., Jr. Midpalatal suture maturation: Classification method for individual assessment before rapid maxillary expansion. Am. J. Orthod. Dentofac. Orthop. 2013, 144, 759-769. [CrossRef]

10. Salmoria, I.; de Souza, E.C.; Furtado, A.; Franzini, C.M.; Custodio, W. Dentoskeletal changes and their correlations after microimplant-assisted palatal expansion (MARPE) in adults with advanced midpalatal suture ossification. Clin. Oral Investig. 2021, 1-11. [CrossRef]

11. Shetty, S.K. Surgically Assisted Rapid Palatal Expansion (SARPE): A Literature Review. Sch. J. Dent. Sci. 2021, 8, 25-40. [CrossRef] 
12. Mohan, A.; Shanthasundari, K.K. Ashwin George Mathew, And Arthi Balasubramaniam. Bone Density Assessment of Midpalatal Suture Using Grayscale Values-A Cbct Study. Eur. J. Mol. Clin. Med. 2021, 8, 2021.

13. Knigge, R.P.; McNulty, K.P.; Oh, H.; Hardin, A.M.; Leary, E.V.; Duren, D.L.; Valiathan, M.; Sherwood, R.J. Geometric morphometric analysis of growth patterns among facial types. Am. J. Orthod. Dentofac. Orthop. 2021, 160, 430-441. [CrossRef] [PubMed]

14. Moon, H.-W.; Nam, W.; Ahn, H.-W.; Oh, S.H.; Kook, Y.-A.; Kim, S.-H. Development of a maxillomandibular arch form based on the center of resistance of teeth using cone-beam computed tomography. Am. J. Orthod. Dentofac. Orthop. 2021, 161, 208-219. [CrossRef]

15. Feragalli, B.; Rampado, O.; Abate, C.; Festa, F.; Stromei, F.; Caputi, S.; Guglielmi, G.; Macrì, M. Cone beam computed tomography for dental and maxillofacial imaging: Technique improvement and low-dose protocols. Radiol. Med. 2017, 122, 581-588. [CrossRef]

16. Blum, R.W.; Astone, N.M.; Decker, M.R.; Mouli, V.C. A conceptual framework for early adolescence: A platform for research. Int. J. Adolesc. Med. Health 2014, 26, 321-331. [CrossRef]

17. Festa, F.; Rotelli, C.; Scarano, A.; Navarra, R.; Caulo, M.; Macrì, M. Functional Magnetic Resonance Connectivity in Patients with Temporomadibular Joint Disorders. Front. Neurol. 2021, 12, 629211. [CrossRef]

18. Holzinger, D.; Carvalho, P.H.D.A.; dos Santos, J.C.; Wagner, F.; Gabrielli, M.A.C.; Gabrielli, M.F.R.; Filho, V.A.P. Bone formation after surgically assisted rapid maxillary expansion: Comparison of 2 distraction osteogenesis protocols. Oral Surg. Oral Med. Oral Pathol. Oral Radiol. 2021, 33, 3. [CrossRef]

19. Chae, J.-M.; Rogowski, L.; Mandair, S.; Bay, R.C.; Park, J.H. A CBCT Evaluation of Midpalatal Bone Density in Various Skeletal Patterns. Sensors 2021, 21, 7812. [CrossRef]

20. Lione, R.; Franchi, L.; Fanucci, E.; Laganà, G.; Cozza, P. Three-dimensional densitometric analysis of maxillary sutural changes induced by rapid maxillary expansion. Dentomaxillofacial Radiol. 2013, 42, 71798010. [CrossRef]

21. Fastuca, R.; Michelotti, A.; Nucera, R.; D’Antò, V.; Militi, A.; Logiudice, A.; Caprioglio, A.; Portelli, M. Midpalatal Suture Density Evaluation after Rapid and Slow Maxillary Expansion with a Low-Dose CT Protocol: A Retrospective Study. Medicina 2020, 56, 112. [CrossRef] [PubMed]

22. Antoun, J.S.; Mei, L.; Gibbs, K.; Farella, M. Effect of orthodontic treatment on the periodontal tissues. Periodontol. 2000 2017, 74, 140-157. [CrossRef] [PubMed] 